\title{
Blockchain Disruption on Management Accountant's Role: Systematic Literature Review
}

\author{
Vecco Suryahadi Saputro \\ Faculty of Economics and Business \\ Universitas Padjadjaran \\ Hamzah Ritchi \\ Faculty of Economics and Business \\ Universitas Padjadjaran \\ Sofik Handoyo \\ Faculty of Economics and Business \\ Universitas Padjadjaran
}

\begin{abstract}
Blockchain considered as an emerging technology that potentially disrupts how management accountant work and his role. This research intends to understand what Blockchain's capabilities will disrupt the profession by conducting a systematic literature review (SLR). A protocol of SLR is inspired by Kitchenham, Van Akthley, and Okoli in their works. The protocol consists of identifying purpose and outcome research, definition research question, preparing search and review protocol, title and abstract screening, paper quality assessment, data extraction, and data analysis. MAXQDA was used to conduct the SLR protocol. Seven academic journal databases were used in the searching stage. Kitchenham's guidelines inspire data extraction and narrative synthesis. This study finds that Blockchain enables the user to do real-time accounting, gather data for supervising and monitoring function, and streamline the accounting practice process. With these findings, the management accountant can prepare to blockchain disruption by upgrading his analytics and computation skills.
\end{abstract}

Keywords: Blockchain; Management accountant; Systematic literature review; MAXQDA

\section{Introduction}

The Current transaction system is often centralised and maintained by the third party. For example, a digital transaction between individuals or companies required banks or credit card providers as a middleman to complete the transaction (Yli-Huumo, Ko, Choi, Park, \& Smolander, 2016a). Impact of a centralised system is middleman cost, data or information integrity issue, and data privacy (Nair \& Sebastian, 2017). Blockchain is emerging and disruptive technology that will be a solution to a centralised transaction system problems. Blockchain is distributed ledger technology that all authorised members in the same network share transaction information between parties (Yoo, 2017). Blockchain maintains a continuous data record verified by all authorised members in the same networks (Yli-Huumo et al., 2016a; Yoo, 2017). In that way, Blockchain enables transparent and more secure data record than a centralised transaction system.

There is a massive trend in blockchain implementation and research about this emerging technology. In 2018, there are 1,140 companies in North America, Europe, and Asia that started the trial-error implementation of blockchain technology (Friedlmaier, Tumasjan, \& Welpe, 2018). Also, 140 blockchain trial reports conduct by energy companies in 2019 (Andoni et al., 2019). This trend shows us that 
Blockchain is a topic that caught the company's and academic's interest. This interest came from its potential recognised by companies and exciting topics for academics. Blockchain can reduce verification and intermediaries cost (Andoni et al., 2019) and reform the financial market and supply chain model (Walport, 2015; Yli-Huumo, Ko, Choi, Park, \& Smolander, 2016b).

Blockchain also can disrupt how the management accounting profession works (Moll \& Yigitbasioglu, 2019a). It happens because of the implementation of new technology and technology change, which significantly impacts the management accountant's role (Pietrzak \& Wnuk-Pel, 2015a). In this case, Blockchain is emerging and disrupting technology that will be faced by a management accountant. Only 20\% of decision-makers in financial companies and $80 \%$ of the 2017 global benchmarking survey respondents know that Blockchain is (Equisoft, 2017; Hileman \& Rauchs, 2017). Most finance professionals lack the understanding of blockchain results in the birth of new profession specifically-aiming to address the issue of Blockchain in finance and accounting (Dutta \& Lawson, 2009; Moll \& Yigitbasioglu, 2019a). There is pressure on academics and professionals to understand more about Blockchain and how it will be disrupted.

In this study, the author focuses on blockchain potential that will disrupt the management accountant's role. The author uses a systematic literature review to gather and analyse journal article that studies Blockchain and management accountant. Purpose of this study is to reveal Blockchain that potentially disrupts management accountants. This study will be organised as follows: Section 2 literature review of blockchain technologies, management accountant's roles, and concept of disruptive technologies, Section 3 describes the process of the systematic literature review (SLR), Section 4 describe findings of SLR in descriptive and statistic, Section 5 and 6 concludes the paper and discuss future studies.

\section{Literature Review}

\section{Overview of Blockchain Technology}

Many studies define Blockchain as distributed ledger technologies. In general, Blockchain is DLT that enables us to have a distributed peerto-peer network that involves confirmation and verification of data blocks (Yoo, 2017). Blockchain is systems that distributed a timestamped data block to every authorised user in the blockchain network (Cao, 2017). In his report, Deloitte revealed that Blockchain is a particular case of DLT where consensus protocol makes chained immutable, and dispute ledger of all transaction shared in the network members (Deloitte, 2017). In conclusion, Blockchain is defined as a distributed database system of continuously and immutable growing data or transaction records verified by authorised Blockchain members.

Blockchain has several advantages. First, Blockchain emerges to create a decentralised environment that does not need a third party as intermediaries or controllers of transaction and data (Yli-Huumo et al., 2016a). Second, data security and privacy create multisignature protection or distributed data records (Kshetri, 2017). Third, Blockchain provides the user to use data in real-time (Drosatos \& Kaldoudi, 2019). Several benefits may potentially disrupt when companies or organisations implement a blockchain, such as disintermediation,non-repudiation, automation, streamlined process, processing speed, cost reduction, and trust (Hughes et al., 2019).

\section{Management Accountant's Role}

In principle, a management accountant's primary roles are decision making, planning, and controlling (Garrison, Noreen, \& Brewer, 2012). In organisations, management accountant involves in strategic decision making and planning by providing futurelooking information and taking part in daily operational decision making (Morales \& Lambert, 2013; Taipaleenmäki \& Ikäheimo, 2013). The controlling role of the accountant includes activities including process gathering, 
evaluating, and responding to examine an operation's compliance (Belfo \& Trigo, 2013), conducting budgetary control (Aziz, Puteh, \& Rahman, 2010), and evaluating organisation performance (Janin, 2017). IMA reported that the management accountant's roles could be described as a business partner that involves decision-making, devising planning and performance management systems, and providing financial reporting expertise (IMA, 2019). Management accountant also becomes an information analyst and data interpreter that helps companies know its performance (Pietrzak \& Wnuk-Pel, 2015b; Vakalfotis, Ballantine, \& Wall, 2011).

However, the roles will evolve as impacts of business environment dynamism and technological advances (Damasiotis, Trivellas, Santouridis, Nikolopoulos, \& Tsifora, 2015). In his works, Pietrzak mentioned that technology development is an external factor that impacts the management accountant's roles (Pietrzak \& Wnuk-Pel, 2015b). Technology development change collection, process, and interpret the data or information that useful to management accountant. To make his/her roles relevant to advances in technologies, management accountants should develop his/her skills and knowledge of emerging technologies (Parker, 2002; Tanaka \& Sithole, 2015). For example, analytical/technical, system knowledge, and interpretation data from the information system are few management skills.

\section{Disruptive Technologies}

Disruptive technologies emerge in the new market because its less risky than entering an established market (Christensen, 1997). In other, disruptive technologies offer something new and better than sustainable innovation. There is an explanation of three elements of the failure framework that become the basis of his disruptive technologies. Emerging of disruptive technologies can be explained by a layer model proposed in Kilkki's studies. In his model, disruption effects through agents and multiple layers (Kilkki, Mäntylä, Karhu, Hämmäinen, \& Ailisto, 2018). A disruption started from the scientific community layer by the discovery of theory. Research and development unit of companies and public organisations begin to create technical inventions based on an accumulation of theory found by the first agent. Firm allocating resources is to develop the technical inventions to a commercially successful invention. After that, the effect of disruption diffuses through multiple layers like industries, consumers, and society/authorities.

There are Three elements of disruptive technologies found by Christensen. First, disruptive technologies are different from sustaining technologies. Second, the development of technologies is overshoot customer or market needs. Finally, leader market companies are spending their investment that appears to be attractive (Christensen, 1997). Other researchers had studied disruptive technology in Christensen work. In Vecchiato works, disruptive technologies enable new features that will be inferior to mainstream features that are particularly important to mainstream customers (Vecchiato, 2017). Emerging of disruptive technologies can impact to performance dimension that can change the base of competition between companies (Danneels, 2004; Li, Porter, \& Suominen, 2018).

Blockchain can be identified as disruptive technologies because of its capabilities to change competition between companies. Also, Blockchain enables new features for some industries and companies. For example, Blockchain enables new features to prevent adverse behaviour and repercussions when implemented in a financial instrument (Lindman, Tuunainen, \& Rossi, 2017). Companies' strategies would adopt these new features on financial instruments. Most studies report that management accountant is seen as a profession that susceptible to disruptive technologies. Most role and task of management accountant are influence by emerging technologies and implementation of information technology system (Pietrzak \& Wnuk-Pel, 2015b). Also, management accountant qualities depend on how they use IT tools to gather and process raw data. Nevertheless, there is the possibility of failure 
to adopt technological change in accounting education (Dutta \& Lawson, 2009). Impact of these is a new discipline that emerges outside the discipline of accounting that disrupts jurisdictions of management accountant and other accounting professions (Moll and Yigitbasioglu 2019).

\section{Methods}

The systematic literature review was selected as the research methods of this research study, a protocol of this study inspired by Chitu Okoli and Schabram work. The researcher also follows guidelines from Kitchenham and Charters to search and to screen relevant papers. The process for systematic literature review is presented in Figure 1.
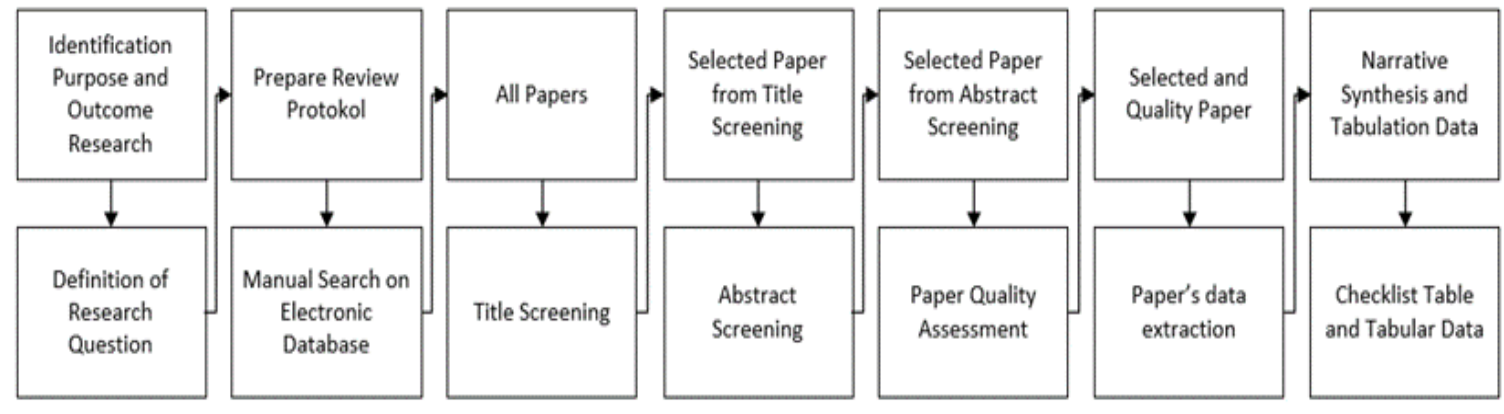

Figure 1. Systematic literature review's process

The researcher selected systematic literature as a research method because it explores blockchain disruption to management accountant role through an existing study. This systematic literature review would help the reader identify and map research areas related to blockchain technology.

\section{Research questions formulation}

The first stage of a systematic literature review is the formulation of the research question (Kitchenham et al., 2009; Okoli, 2015; YliHuumo et al., 2016b). The goal of this research was to provide an in-depth view of disruption blockchain to management accountant. Therefore, the researcher determines two research questions:

RQ1: What are the latest research focusing on blockchain disruption concerning management accountant profession?

RQ2: How is blockchain application disrupt management accountant role in an organisation?

\section{Conducting Search Strategy}

The second step of this research is to prepare and conduct a search strategy. A search protocol must define how researchers search for all relevant papers and studies. This protocol aims to minimise the possibility of research bias (Yli-Huumo et al., 2016b). Researchers choose to focus on peer-reviewed papers published in conference proceedings and journals. We used IEEEXplore, ACM Digital Library, SpringerLink, ScienceDirect, PLOSOne, AISEL, and JSTOR as journal databases. Combination keywords and Boolean were used to search for studies through journal databases. The Boolean operators used were restricted to AND and OR. The combination keywords were: ("Blockchain" OR "Blockchain application") AND ("Management Accountant" or "Accountant").

\section{Title and Abstract Screening}

Not all papers and studies are relevant to this research question. They needed to be assessed for their relevance by first screening 
(Kitchenham et al., 2009; Taylor, Dargahi, Dehghantanha, Parizi, \& Choo, 2019). This stage consists of title screening and abstract screening. Inclusion and exclusion criteria were defined for this screening. The essential inclusion and exclusion criteria are shown in Table 1.

Table 1. Inclusion and exclusion criteria

\section{Criteria for inclusion}

1. The studies must be relevant to Blockchain Application and its effect on Management Accountant.

2. The studies must be a peer-reviewed product that publishes between 2008 2019.

\section{Criteria for Exclusion}

1. Non-English language papers.

2. Incomplete paper that does not have exact abstract and full text

3. Paper that does not mention blockchain and management accountant role.

\section{Quality Assessment}

A quality assessment of primary studies must be conduct to select the more relevant papers to the research question (Kitchenham et al., 2009; Taylor et al., 2019). In this study, quality assessment was inspired by Dyba and Dingsory research that contain 11 item criteria (Dybå \& Dingsøyr, 2008). The 11 criteria covered four main issues: reporting, rigour, credibility, and relevance. Each item would be scored as zero (no) and one (yes).

\section{Data extraction and analysis}

Next stage is extracting data from papers that passed the quality assessment and first screening. Data extraction was coded as D0D11. D0-D07 gathers necessary information of papers. D08-D11 was extracted by reading the papers. Researchers collected the extracted data by MAXQDA and presented by Excel. Data extraction item and its code are shown in Table 2.

Table 1. Item of the data extraction process

\begin{tabular}{ll}
\hline Code & Data Item \\
\hline D0 & Study identification \\
\hline D01 & Title \\
\hline D02 & Authors \\
D03 & Country \\
D04 & Publication info \\
D05 & Publication type \\
D06 & Publication source \\
D07 & Publication year \\
\hline D08 & Focus research \\
D09 & Research goal \\
D10 & Research question \\
D11 & Design study and methodology \\
\hline D12 & Result \\
\hline
\end{tabular}

After the extraction data stage, the next process was analysing with narrative synthesis. The reason for choosing this synthesis is its capability to collect and analyse data research from qualitative or quantitative methodology
(Cochrane, 2004). In this study, the synthesis process based on works of Keathley \& Van Aken and Popay. The first step was collecting and making tabulation based on each paper's findings (Keathley \& Aken, 2013). The second 
step, we categorised all factors and disruption potential. The last step was calculating frequency each factor that mention by studies and papers

\section{Results}

\section{Search and selection result}

This section results from a search, first screening, and quality assessment from the systematic literature review protocol. The search and selection result is presented in Figure 2. 272 papers collected after conducting the search stage and duplication removal. All papers were screening by its abstract and title separately, which led to 123 papers combined. The excluded paper's reason was that the title and abstract were not mentioned blockchain disruption to management accountant.

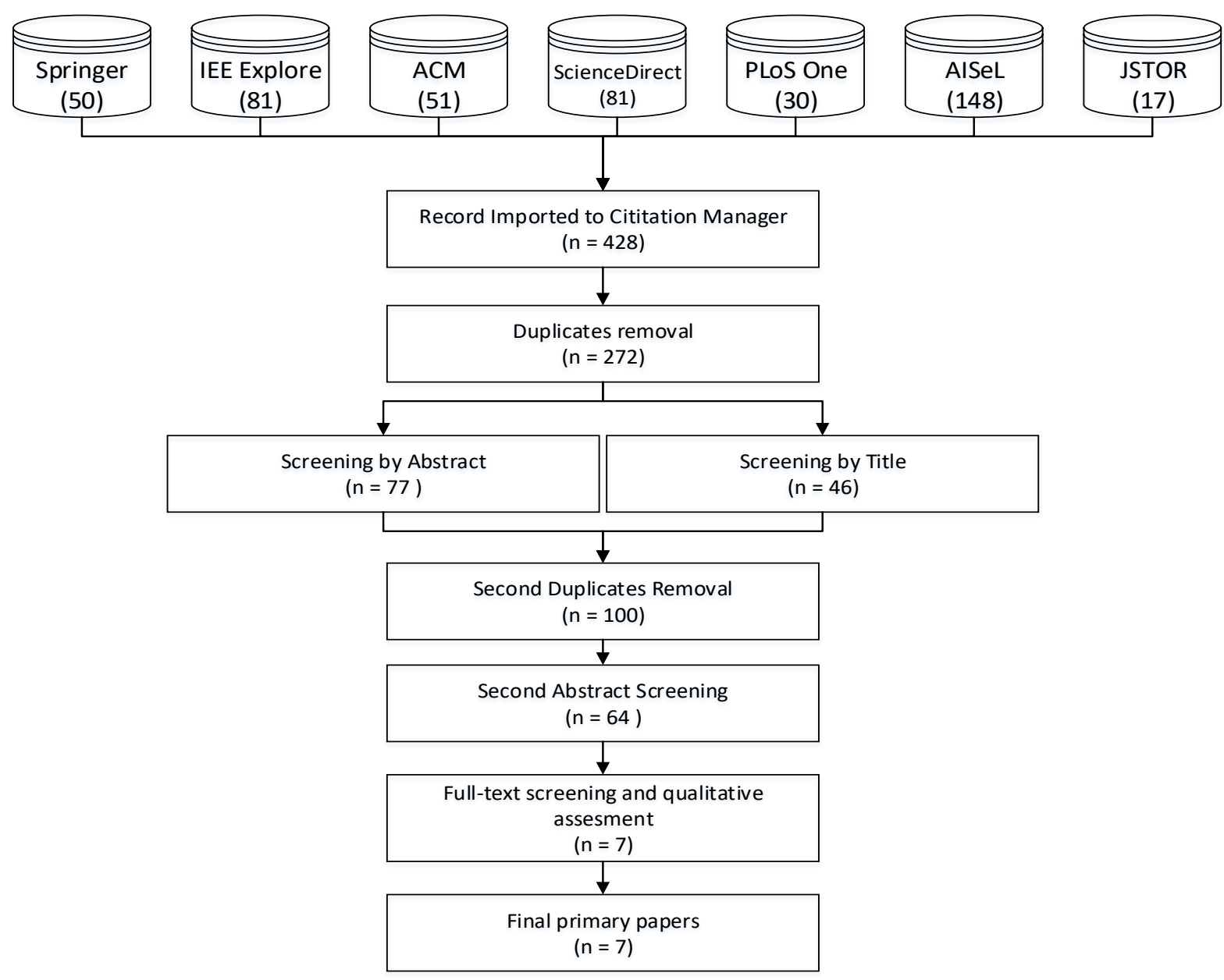

Figure 2. Flowchart of systematic literature review

After selecting 123 papers, we removed duplication and done second abstract screening that divined by inclusion and exclusion criteria. The result of the second abstract screening is 64 papers. After that, the researcher has done fulltext screening with a qualities assessment item. Only seven papers that pass qualities assessment, which would be used for extraction and analysing stage. The reason excluded papers (57) was that their full-text was not available, do not mention blockchain disruption and management accountant and do not pass QA 1-3. The seven papers coded, as shown below. 
Table 3. Code and title of primary studies

\begin{tabular}{|c|c|}
\hline Code & Title \\
\hline R1 & Automatic Information Disclosure with Value Chains Based on Blockchain Technology \\
\hline $\mathrm{R} 2$ & Blockchain's roles in strengthening cybersecurity and protecting the privacy \\
\hline R3 & $\begin{array}{l}\text { The role of internet-related technologies in shaping the work of accountants: New } \\
\text { directions for accounting research }\end{array}$ \\
\hline R4 & Blockchain and the built environment: Potentials and limitations \\
\hline R5 & A Blockchain Research Framework \\
\hline R6 & LoC - A new financial loan management system based on smart contracts \\
\hline $\mathrm{R} 7$ & $\begin{array}{l}\text { Application of Internet of Things and Blockchain Technologies to Improve Accounting } \\
\text { Information Quality }\end{array}$ \\
\hline
\end{tabular}

\section{Distribution of meta-data papers}

This research process RIS of each primary study was conducted by software MAXQDA 2018 to gather data of publication year, type of primary studies, geographical distribution researcher, and publication channel. Results of this process are to answer RQ 1. Figure 3 shows the publication year distribution of primary studies. Figure 4 shows us the type of primary studies. Figure 5 show us, geographic distribution researchers. Table 3 shows the publication channel of selected primary papers.

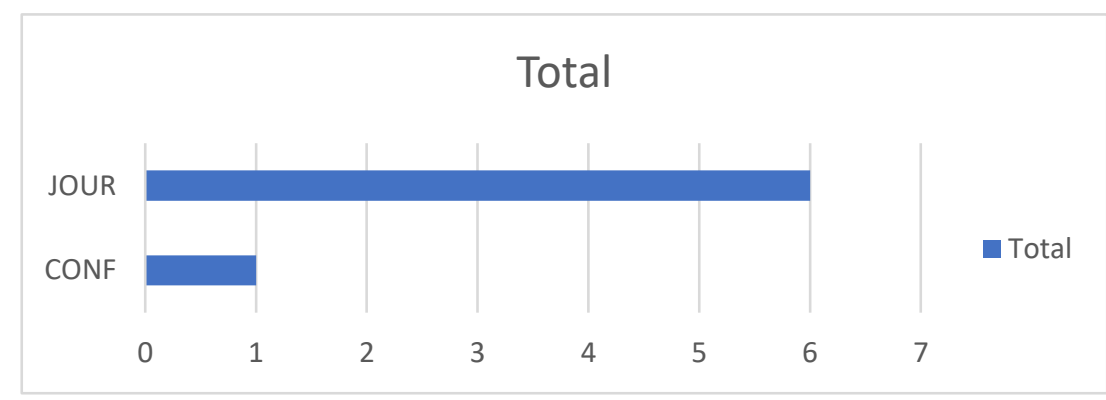

Figure 1. Type of publication of primary studies

Two papers (29\%) is Published in 2017, one paper (14\%) is published in 2018, and four papers $(57 \%)$ is published in 2019. It shows that Blockchain and disruption to management accountant are a recent topic. The reason behind this trend is the idea of Blockchain was found and studied after its white paper published in 2008 (Moll \& Yigitbasioglu, 2019a; YliHuumo et al., 2016b).

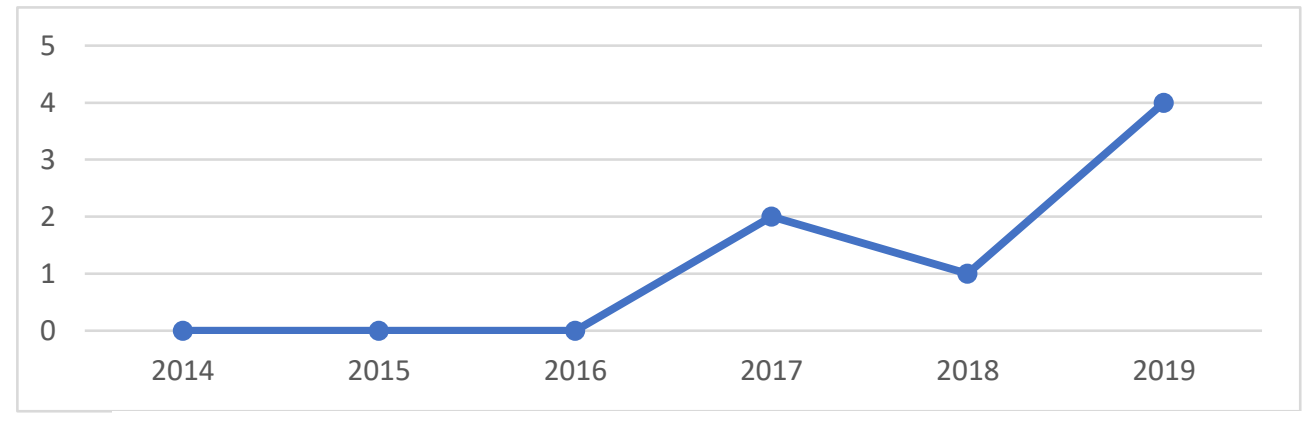

Figure 2. Distribution publication year 
In Figure. 4, all primary studies were divided into journal and conference. Six papers $(867 \%)$ are a journal article, and one paper $(14 \%)$ are conference proceedings. These come from a systematic literature review's criteria that only journal article and conference proceedings can be used as primary studies. Grey literature did not include in these studies because peer or reviewers have not reviewed it.

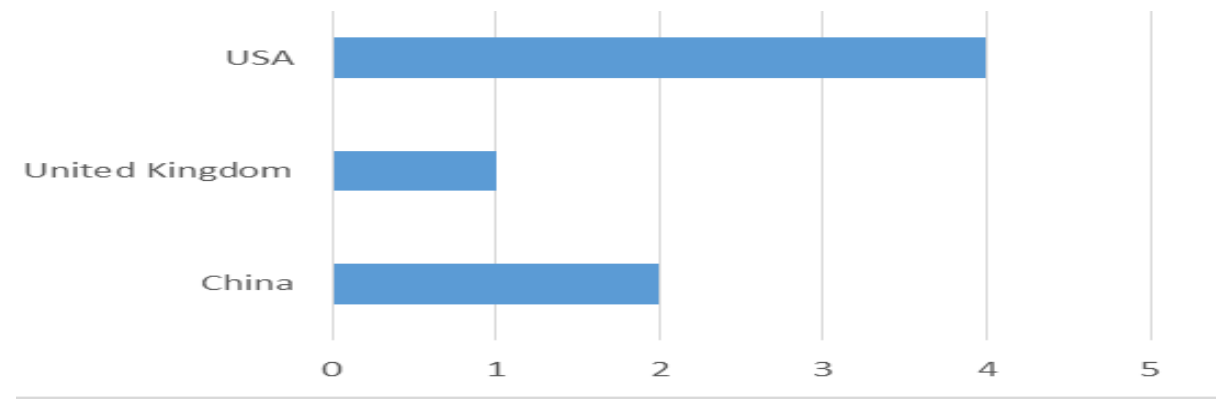

Figure 3. Distribution of researcher's country

Figure 5 show us that blockchain researcher originated from China (29\%), United Kingdom (14\%), and USA (57\%). In table 4, primary studies were distributed to seven publication channels. IEEE ITAIC 2019, IEEE Access, and Business \& Information System Engineering are a publication channel that publishes research on information technology, artificial intelligence, and information system. Future Generation Computer Systems provide articles that study advances in a distributed system, collaborative environment, high-performance computing, and technology. Journal of Building Engineering provides a journal covering all aspects of science and technology concerned with the built environment's whole life cycle. Telecommunication Policy is concerned with the impact of digitalisation in economy and society. The British Accounting Review publish a paper on accounting and finance as a research focus.

Table 4. Publication channel and codes of primary studies

\begin{tabular}{l|c}
\hline Publication Channel & Code \\
\hline $\begin{array}{l}\text { 2019 IEEE 8th Joint International Information Technology and Artificial Intelligence } \\
\text { Conference (ITAIC) }\end{array}$ & R1 \\
\hline Business \& Information Systems Engineering & R5 \\
\hline Future Generation Computer Systems & R6 \\
\hline IEEE Access & R7 \\
\hline Journal of Building Engineering & R4 \\
\hline Telecommunications Policy & R2 \\
\hline The British Accounting Review & R3 \\
\hline
\end{tabular}

\section{Findings}

This section explains blockchain capabilities and potential that disrupt the management accountant's role. In this section, the researcher gathers data by reading selected primary studies, then coded sentence or paragraph that contain disruption potential into codes by used MAXQDA 2018. Each code would be count its frequencies. Result of these findings shown in Table 4.

Table 5 is a table that shows the frequency of blockchain disruption capabilities that have been mention by primary studies. These disruption capabilities already categorised from each finding. The research showed that three significant disruption 
potential to management accountant are Blockchain enables real-time accounting $(71,43 \%)$, information available for analysing function $(57,14 \%)$ and improve efficiencyeffectiveness accounting practice $(57,14 \%)$.

Table 2. Frequency of blockchain disruption potential

\begin{tabular}{llll}
\hline Blockchain Disruption Potential & Frequency & Percentage & Studies \\
\hline Enable real-time accounting & 5 & 71.43 & $\begin{array}{l}\text { R1, R3, R4, } \\
\text { R6, R7 }\end{array}$ \\
$\begin{array}{l}\text { Information available for analyze/supervise } \\
\text { function }\end{array}$ & 4 & 57.14 & $\begin{array}{l}\text { R1, R2, R3, } \\
\text { Improve efficiency and effectiveness of }\end{array}$ \\
$\begin{array}{l}\text { accounting practice } \\
\text { Support risk mitigation }\end{array}$ & 4 & 57.14 & R1, R3, R5, \\
Enable self-verification transaction/data & 3 & 42.86 & R6 \\
Enable continuous monitoring & 2 & 42.86 & R3, R6, R6 \\
\hline $\begin{array}{l}\text { Automated distribution of information } \\
\text { Real-time access to performance data }\end{array}$ & 2 & 28.57 & R1. R6 \\
\hline Increase accuracy and reliability & 1 & 28.57 & R1, R7 \\
Compliance to standard & 1 & 14.29 & R3 \\
Decreasing transmission cost & 1 & 14.29 & $\mathrm{R} 7$ \\
\hline Improving corporate governance level & 1 & 14.29 & $\mathrm{R} 7$ \\
\hline Allows transactional reporting & 1 & 14.29 & $\mathrm{R} 7$ \\
\hline Tracking error information & 1 & 14.29 & $\mathrm{R} 7$ \\
\hline Documents with code (s) & 7 & 14.29 & $\mathrm{R} 3$ \\
\hline
\end{tabular}

Enable users to real-time accounting. Blockchain enables users to real-time accounting. This mean management accountants as users can access transaction and other data in real-time and on-demand (Moll and Yigitbasioglu, 2019; Wang, Guo and Cheng, 2019; Wu, Xiong and Li, 2019). This capability could be accelerating the shift from a periodical to instantaneous information for decision-makers (Nawari \& Ravindran, 2019; Wu et al., 2019). This capability also enables to update and record transaction data immediately (Cai, Xu, \& Zhang, 2019; Nawari \& Ravindran, 2019).

The information available for analysing/supervise the function. Information on the Blockchain is available in real-time for the user, such as inquisitor (Kshetri, 2017), stakeholder, and management accountant (Moll and Yigitbasioglu, 2019). With the information shared in Blockchain, stakeholders can retrieve disaggregated information to report and financial statements based on their needs. Management accountant can analyse and give support to information users by providing financial and non-financial information.

Improve efficiency and effectiveness of accounting practice. With the blockchainaccounting systems, immutability records and transaction data are shared and link in authorised users (e.g. trading partners, suppliers, and auditors). This capability enables management accountants as the user to improve information integrity, increase the speed of transaction settlement, and prevent a fraudulent transaction (H. Wang et al., 2019). The impact of these capabilities is improving on the efficiency of accounting practice, decreasing transmission cost, and increasing assurance (Moll and Yigitbasioglu, 2019). Blockchain system would improve internal control, risk mitigation, and prevent fraudulent transactions with its capability to maintain irreversible records. 
Enable self-verification. Dai and Vasarhelyi proposed a "triple-entry" bookkeeping approach based on blockchain application (Moll \& Yigitbasioglu, 2019c; Wu et al., 2019). This approach enables all record transaction to be verified with Blockchain as independent and reliable parties. This approach's result is three entries (debit, credit, and cryptographic signature) to verify transaction or data.

Continuous monitoring. With its distributed transaction and activity record database, Blockchain enables management accountant to continuously monitor the flow of asset or transaction (Cai et al., 2019; Y. Wang $\&$ Kogan, 2018). It happens because of the realtime availability of transaction data and realtime accounting that enabled by blockchain technology. Impact of this continuous monitoring is preventing managers from doing earning management and commit fraud.

Automated distributed information. As distributed ledger technology, information on the blockchain network is shared with other nodes or organisation (Cai et al., 2019; Wu et al., 2019). In Wang's BB-TPS work, transaction data are automatically distributed to authorised managers listed in blockchain networks (Y. Wang \& Kogan, 2018). These data become internal information of an enterprise.

Real-time performance access data. Blockchain also gives a chance to management accountant to read and analyse company performance data at any time. Impact of these capabilities is that the user can recommend immediate corrective decisions (Moll and Yigitbasioglu, 2019).

Increase accuracy and reliability. Blockchain made information and data cannot be tampered and irreversible. Once a transaction record in the network, it is hard to manipulate or change the data. Blockchain can provide the user with this capability to reliable accounting information (Wu et al., 2019). Accuracy data can be achieved by integrating Blockchain with IoT Technology or RFID that enable real-time and automatically updating of information, such as inventory information.

Compliance to standards. In the proposed BC-IoT, smart contracts based on blockchain technology ensure the compliance of the accounting information process (Wu et al., 2019). By smart contract, the standard of processing accounting information is described as a contract. If there is no contract, then there is no transaction.

Decreasing transmission cost. Blockchain gives opportunities to the transaction processing system to records transaction and other data with high integrity and low transmission cost. In Wang's case, transmission cost defines as a cost for transmitting data (Y. Wang \& Kogan, 2018). Also, in his case, the transmission cost of Blockchain was compared to a contemporary database. This function help management accountant to reduce unnecessary cost.

Improving corporate governance. Improving corporate governance level is the impact of the truthfulness of financial information, compliance to standards, and increasing reliability of the information that enabled by blockchain technology (Wu et al., 2019). The most critical task for management accountants is to provide truthful and reliable information for decision making.

Tracking error information. In a proposed integrated value chain system based on Blockchain, the system enabled management accountant to find the evidence of its error and distortion information from the source (Cai et al., 2019).

\section{Discussion}

The purpose of this study is to reveal blockchain benefits and capabilities that potentially disrupt the management accountant's role and task. A research set research question seeks information about the latest and distribution research about disruption blockchain to management accountants. To compete for this purpose, the researcher used a systematic literature review as a 
methodological approach because it enables researchers to seek current studies. This research finds that most studies related to blockchain disruption to management accountant were published in 2019. These findings show us that blockchain disruption in management accountant is a relatively new topic in accounting academics. This research also finds that most blockchain capability that potentially disrupts the management accountant's role enables real-time accounting consistent with a report published by AICPA and IMA. Other potential capabilities consistent with the reports likes' information are available for monitoring and supervise function and improving the efficiency of accounting practices.

Blockchain's capabilities have a potential impact on the management accountant profession. First, the management accountant can provide real-time data for planning and controlling process. Second, the management accountant enables us to do continuous monitoring to ensure strategy and plan execution. Third, manual and routine tasks can be reduced by Blockchain. Fourth, the management accountant can provide more reliable and accurate information for decision making. There are two limitations to this study. First, there are limited numbers of the database for identifying potentially eligible studies; the Greater number of primary studies can be generalised the conclusion. Second, there is potential bias risk from researcher or interpretation of primary studies. The use of triangulation can solve this limitation. Further research is expected to use many journal databases, conduct triangulation, and verify the blockchain potential with other methodology.

\section{Conclusion}

There are three findings in this study. First, Blockchain as disruption technology could expand the management accountant's role and help conduct some tasks. For example, three most blockchain disruption potential factors enable real-time accounting, providing information to monitor and supervise performance's organisation and improve efficiency and effectiveness in accounting practice. Second, this study found few studies on how Blockchain disrupt management accountant as a profession. Third, the topics of Blockchain and management accountant are relatively novel in accounting and business academics.

Although this study chooses to implement rigour systematic literature review's protocol, there is always a bias risk from a researcher or interpretation of primary studies. For the subsequent studies, it is expected to conduct triangulation to reduce bias risk. It can be done by researcher triangulation or method triangulation. To get an in-depth understanding, researchers interested in continuing on this topic can re-searching these findings by conducting other methods, such as interview and survey.

\section{References}

Andoni, M, V. Robu, D. Flynn, S. Abram, D. Geach, D. Jenkins, P. McCallum, and A. Peacock. 2019. "Blockchain Technology in the Energy Sector: A Systematic Review of Challenges and Opportunities." Renewable and Sustainable Energy Reviews 100 (November): 143-174.

Aziz, R., C. Hamidah Che Puteh, and A. Zaliha Abdul Rahman. 2010. "Bridging Theory and Practice: Lessons on Management Accounting from Selected Practices in Malaysia." In 2010 International Conference on Science and Social Research (CSSR 2010), 53-57.

Belfo, F., and A. Trigo. 2013. "Accounting Information Systems: Tradition and Future Directions." Procedia Technology 9: 536-456.

Cai, S., M Xu, and L Zhang. 2019. "Automatic Information Disclosure with Value Chains Based on Blockchain Technology." In 2019 IEEE 8th Joint International Information Technology and Artificial Intelligence Conference (ITAIC), 1534-1538.

Cao, L. 2017. "Behavior Informatics to Discover Behavior Insight for Active and Tailored Client Management." In Proceedings of the 23rd ACM SIGKDD International 
Conference on Knowledge Discovery and Data Mining, 15-16.

Christensen, C. 1997. The Innovator's Dilemma. President and Fellows of Harvard College. Boston.

Cochrane. 2004. "Reviewers' Handbook," no. March.

Damasiotis, V., P. Trivellas, I. Santouridis, S. Nikolopoulos, and E. Tsifora. 2015. "IT Competences for Professional Accountants. A Review." Procedia - Social and Behavioral Sciences 175 (February): 537-545.

Danneels, E. 2004. "Disruptive Technology Reconsidered: A Critique and Research Agenda." Journal of Product Innovation Management 21 (1): 246-258.

Deloitte. 2017. "Blockchain Technology and Its Potential Impact on the Audit and Assurance Profession."

Drosatos, G., and Eleni K.. 2019. "Blockchain Applications in the Biomedical Domain: A Scoping Review." Computational and Structural Biotechnology Journal 17: 229-240.

Dutta, S., and R. A. Lawson. 2009. "Analysis of Accounting Academe's Response to Structural Changes in the Profession Using the Disruptive Technology Framework." International Journal of Critical Accounting 2 (1): 19.

Dybå, T., and T. Dingsøyr. 2008. "Empirical Studies of Agile Software Development: A Systematic Review." Information and Software Technology 50 (9-10): 833-859.

Equisoft. 2017. "Blockchain A Disruptive Technology with the Power to Revolutionise Financial Services."

Friedlmaier, M., A. Tumasjan, and I. M. Welpe. 2018. "Disrupting Industries with Blockchain: The Industry, Venture Capital Funding, and Regional Distribution of Blockchain Ventures." Proceedings of the 51st Hawaii International Conference on System Sciences, no. July: 3517-3526.

Garrison, R., E. W. Noreen, and Peter C. Brewer. 2012. Managerial Accounting. Edited by Stewart Mattson. 14th ed. New York, NY, USA: McGraw-Hill Irwin.

Hileman, G., and M.l Rauchs. 2017. "Global Blockchain Benchmarking Study." Cambridge Centre for Alternative Finance. Cambridge.

Hughes, L., Y. K Dwivedi, S. K Misra, N. P Rana, V. Raghavan, and V. Akella. 2019. "Blockchain Research, Practice and Policy: Applications, Benefits, Limitations, Emerging Research Themes and Research Agenda." International Journal of Information Management 49 (February): 114-129.

IMA. 2019. "Ima Management Accounting Competency Framework."

Janin, F.. 2017. "When Being a Partner Means More: The External Role of Football Club Management Accountants." Management Accounting Research 35: 5-19.

Keathley, H., and E. Aken. 2013. "Systematic Literature Review on the Factors That Affect Performance Measurement System Implementation," 837-847.

Kilkki, K., M. Mäntylä, K. Karhu, H. Hämmäinen, and H. Ailisto. 2018. "A Disruption Framework." Technological Forecasting and Social Change 129 (September): 275-284.

Kitchenham, B., O. Brereton, D. Budgen, M. Turner, J. Bailey, and S. Linkman. 2009. "Systematic Literature Reviews in Software Engineering - A Systematic Literature Review." Information and Software Technology 51 (1): 7-15.

Kshetri, N. 2017. "Blockchain's Roles in Strengthening Cybersecurity and Protecting Privacy." Telecommunications Policy 41 (10): 1027-1038.

Li, M., A. Porter, and A. Suominen. 2018. "Insights into Relationships between Disruptive Technology/Innovation and Emerging Technology: A Bibliometric Perspective." Technological Forecasting and Social Change 129 (September): 285-296.

Lindman, J., V. Tuunainen, and M. Rossi. 2017. "Opportunities and Risks of Blockchain 
Technologies: A Research Agenda." Proceedings of the 50th Hawaii International Conference on System Sciences (2017), 15331542 .

Moll, J., and O. Yigitbasioglu. 2019a. "The Role of Internet-Related Technologies in Shaping the Work of Accountants: New Directions for Accounting Research." The British Accounting Review.

Morales, J., and C. Lambert. 2013. "Dirty Work and the Construction of Identity. An Ethnographic Study of Management Accounting Practices." Accounting, Organisations and Society 38 (3): 228-244.

Nair, G., and S. Sebastian. 2017. "BlockChain Technology Centralised Ledger to Distributed Ledger." International Research Journal of Engineering and Technology (IRJET) 04 (03): 2823-2827.

Nawari, N., and S. Ravindran. 2019. "Blockchain and the Built Environment: Potentials and Limitations." Journal of Building Engineering 25: 100832.

Okoli, C. 2015. "A Guide to Conducting a Standalone Systematic To Cite This Version : A Guide to Conducting a Standalone Systematic Literature Review." Communications of the Association for Information Systems 37: 879910.

Parker, Lee D. 2002. "Parker 2002 Reinventing_the_management_accountant_ma r02," no. March.

Pietrzak, Ż., and T. Wnuk-Pel. 2015a. "The Roles and Qualities of Management Accountants in Organisations - Evidence from the Field." Procedia - Social and Behavioral Sciences 213: 281-285.

Taipaleenmäki, J., and S. Ikäheimo. 2013. “On the Convergence of Management Accounting and Financial Accounting - the Role of Information Technology in Accounting Change." International Journal of Accounting Information Systems 14 (4): 321-348.

Tanaka, S., and M. Sithole. 2015. "Information Technology Knowledge and Skills Accounting
Graduates Need Economics and Finance." International Journal of Business and Social Science 6 (8): 47-52.

Taylor, P., T. Dargahi, A. Dehghantanha, R. Parizi, and K. Choo. 2019. "A Systematic Literature Review of Blockchain Cyber Security." Digital Communications and Networks.

Vakalfotis, N., J. Ballantine, and A. Wall. 2011. "A Literature Review on the Impact of Enterprise Systems on Management Accounting," no. July: 11-12.

Vecchiato, R. 2017. "Disruptive Innovation, Managerial Cognition, and Technology Competition Outcomes." Technological Forecasting and Social Change 116: 116-128.

Walport, M.. 2015. "UK Government DLT Report: Distributed Ledger Technology: Beyond Block Chain." Government Office for Science, 1-88.

Wang, H., C. Guo, and S. Cheng. 2019. "LoC - A New Financial Loan Management System Based on Smart Contracts." Future Generation Computer Systems 100: 648-655.

Wang, Y., and A. Kogan. 2018. "Designing Confidentiality-Preserving Blockchain-Based Transaction Processing Systems." International Journal of Accounting Information Systems 30: $1-18$.

Wu, J., F. Xiong, and C. Li. 2019. "Application of Internet of Things and Blockchain Technologies to Improve Accounting Information Quality." IEEE Access 7: 10009098.

Yli-Huumo, J, D. Ko, S. Choi, S. Park, and K.Smolander. 2016a. "Where Is Current Research on Blockchain Technology?-A Systematic Review." PloS One 11 (10): e0163477.

Yoo, S. 2017. "Blockchain Based Financial Case Analysis and Its Implications." Asia Pacific Journal of Innovation and Entrepreneurship 11 (3): 3 